\title{
MENSURAÇÃO DO DÍMERO D E SUA INFLUÊNCIA EM PACIENTES COM COVID-19
}

\author{
MEASUREMENT OF D DIMMER AND ITS INFLUENCE ON PATIENTS WITH COVID-19
}

Thalita Leite Oliveira, Julia Gonçalves Souza, Antonia Daiane Silva de Lavor e Francisca Alana de Lima Santos

Centro Universitário Doutor Leão Sampaio

\begin{abstract}
RESUMO
A COVID-19, doença causada pelo SARS-CoV2, não afeta somente o sistema respiratório, mas também todos os sistemas do corpo. Pacientes com a doença, especialmente em sua forma mais grave, são mais propensos a apresentarem elevados índices de coagulopatias. Relacionado a isto está o aumento da taxa do Dímero D (DD), produto da degração da fibrina, formado pela ativação da enzima plasmina. Esses pacientes tem uma maior propensão a desenvolver Trombose Venosa Profunda (TVP), Coagulação Intravascular Disceminada (CIVD), entre outros problemas graves relacionados à coagulação. O objetivo do estudo foi conhecer através da literatura a importância da mensuração do Dímero D em pacientes com Covid-19 e como isso influencia na progressão da doença, sendo a pesquisa realizada nos seguintes bancos de dados: PubMed, Scielo, PEDro e Lilacs, utilizando os descritores "COVID-19", "D dimmer" e "Coagulation" e com o operador booleano AND. Foram selecionados artigos publicados entre os anos de 2020 e 2021, sendo encontrados ao todo 182 artigos, porém utilizados 37 para a revisão. Nos artigos selecionados, mostrou-se a importância da análise do Dímero D em pacientes com covid-19, pois seu aumento pode estar associado a gravidade da doença e ao risco de mortalidade. Além do DD, há a importância da análise e mensuração de outros marcadores tromboembólicos para a análise do prognóstico do paciente. de problemas vasculares/coagulação decorrentes da doença. A mensuração do Dímero D é essencial especialmente para os pacientes com Covid-19, pois pode estar associado à gravidade e ao risco de mortalidade por causa da doença.
\end{abstract}

Palavras-chave: Dímero D; COVID-19; Coagulação.

\section{ABSTRACT}

COVID-19 is a disease caused by SARS-CoV2, not only affects the respiratory system, but also all systems in the body. Patients with the disease, especially in the most severe form, are more likely to have high rates of coagulopathies. Related to this is the increase in the rate of D-Dimer (DD), a product of fibrin degradation, formed by the activation of the plasmin enzyme. These patients are more likely to develop Deep Venous Thrombosis (DVT), Discerned Intravascular Coagulation (DIC), among other serious problems related to coagulation. The aim of the study was to know, through the literature, the importance of measuring the D-Dimer in patients with Covid-19 and how it influences the progression of the disease. The research was carried out in the following databases: PubMed, Scielo, PEDro and Lilacs, using the descriptors "COVID-19", "D dimmer" and "Coagulation" and with the Boolean operator AND. Articles published between 2020 and 2021 were selected, with a total of 182 articles being found, but 37 were used for the review. In the selected articles, the importance of analyzing the D-Dimer in patients with covid-19 was shown, its increase may be associated with disease severity and mortality risk. In addition to DD, there is the importance of analyzing and measuring other thromboembolic markers for analyzing the patient's prognosis of vascular problems/coagulation resulting from the disease. The measurement of D-Dimer is especially essential for patients with Covid-19, as it may be associated with the severity and risk of mortality from the disease.

Keyword: D-dimmer; COVID-19; Coagulation. 


\section{INTRODUÇÃO}

A COVID-19 é uma doença infecciosa causada pelo vírus SARS-CoV-2, novo vírus da família SARSCoV. O novo vírus foi observado pela primeira vez em 2019 na cidade de Wuhan, na China, quando casos de uma pneumonia inexplicada foram observadas na cidade. A maioria das pessoas infectadas desenvolvem uma doença respiratória leve a moderada, sem a necessidade de internação e/ou cuidados mais intensivos (WHO, 2021).

A patogênese desta pode ocorrer por meio de dois mecanismos: lesão imunomediada e invasão do tecido através dos receptores da ECA2 - $2^{\mathrm{a}}$ Enzima de conversão da angiotensina - (PILLAI et al, 2020) que está presente no endotélio vascular, tecido renal, cardiovascular e intestinal, gerando uma hiperinflamação, tempestades de citocina, vasculopatias, coagulopatias intravasculares e problemas associados. Para análise do prognóstico desses pacientes, é importante a mensuração de fatores tromboembólicos, como o Dímero D, além do tempo de protrombina, trombocitopenia (HENRY et al, 2020).

De acordo com Gaffney (1980), o Dímero D, ou D Dímero (DD), é o resultado do processo de fibrinólise, gerado através da formação de um coágulo, seguido de uma ativação da enzima plasmina, degradação da fibrina, resultando por último no DD (dois fragmentos $\mathrm{D}$ da fibrina). Ou seja, a presença dele depende diretamente do nível de coagulação e ativação da fibrinólise (ZHANG et al, 2018). Em razão das disfunções das células endoteliais e respostas inflamatórias às infecções virais, que geram uma ativação excessiva da cascata de coagulação e das plaquetas, o Dímero D se eleva, o que aumenta a produção de trombina e consequentes problemas na coagulação pelo desequilíbrio entre os mecanismos homeostáticos pró e anticoagulantes. (ZHANG et al, 2020; SUBRAMANIAM $\&$ SCHARRER, 2018)

Em virtude do que foi explanado, o objetivo desta pesquisa foi conhecer, através da literatura, a importância da mensuração do Dímero D em pacientes com COVID19 e como isso pode influenciar no prognóstico dos pacientes com a doença.

\section{METODOLOGIA}

Trata-se de uma revisão integrativa realizada no período de outubro de 2021. Esta foi construída através da busca de artigos científicos realizada nas bases de dados: PubMed (179), Scielo (3), PEDro (0), Lilacs (0), com os descritores "COVID-19", "D dimmer", "Coagulation" e o operador booleano AND.

Foram inclusos na pesquisa artigos publicados a partir de
2020, estudos concluídos, gratuitos e na íntegra, nas línguas portuguesa, inglesa e espanhola. Foram excluídos da pesquisa revisões de literatura, monografias ou artigos que fugissem do propósito da pesquisa.

Inicialmente foi realizado o levantamento de 182 artigos seguindo os critérios de elegibilidade. Em seguida, foi realizada a leitura abreviada dos resumos para observação da temática, aspectos metodológicos e objetivos. Na sequência, foi feita a leitura na íntegra, selecionando então 37 artigos para a construção desse estudo.

\section{RESULTADOS E DISCUSSÕES}

Produto da degradação da fibrina através do processo da fibrinólise, o Dímero D acima do valor fisiológico, indica um estado de hipercoagulação no sistema vascular do indivíduo, especialmente naqueles com COVID-19. Dos artigos estudados e revisados, há um consenso em relação ao aumento do D Dímero estar relacionado ao agravamento da condição de saúde do paciente com a doença e, em alguns casos, está associado a mortalidade do paciente. A mensuração desse marcador não é específica para COVID-19, pois também se apresenta elevado na Sepse e na SDRA (Síndrome do Desconforto Respiratório Aguda) (FERRER et al, 2020), embora sirva como importante marcador tromboembólico. Moreno e colaboradores, em 2020, recomendaram a monitorização do DD a cada 24-48 horas em pacientes hospitalizados como ferramenta de avaliação de progressão da doença.

De acordo com YU et al (2020), DD $\geq 0,5 \mu \mathrm{g} / \mathrm{mL}$ é considerado anormal e os pacientes apresentam essa alteração em uma forma mais grave da doença. O Comitê de Diretrizes Clínicas do American College of Physicians propôs avaliar o nível do DD através de ajustes de acordo com a idade com o seguinte cálculo: idade x $0,01 \mu \mathrm{g} / \mathrm{mL}$ a fim de entender o valor mais apropriado para cada paciente (RAJA et al, 2020).

Um estudo retrospectivo feito por Zhang e colaboradores, em 2020, em um hospital de Wuhan, China, demonstrou que o $\mathrm{DD} \geq 2,0 \mu \mathrm{g} / \mathrm{mL}$ pode predizer a mortalidade hospitalar dos pacientes diagnosticados com a COVID-19. Os fatores prognósticos da doença estão associados às situações sociodemográficas, histórico patológico do paciente, achados no exame físico, laboratoriais e radiológicos, sendo, no geral, a mortalidade mais alta em pacientes mais velhos e com condições clínicas anteriores (IZCOVICH et al, 2020).

Samkari e colaboradores (2020), constataram em seu estudo multicêntrico em pacientes hospitalizados com 


\section{V.10 N.1 (2022) ISSN: $2317-434 X$}

o diagnóstico da COVID-19, que a incidência de TVE (Tromboembolismo Venoso) confirmado foi maior que a de CIVD (Coagulação Intravascular Disseminada). Além disso, há um alto risco de EP (Embolia Pulmonar) por causa da pneumonia decorrente da doença devido a deposição de fibrina que gera microtrombos nos alvéolos (GIANNIS et al, 2020).

\section{CONCLUSÃO}

A mensuração do Dímero $\mathrm{D}$ é essencial especialmente para os pacientes com COVID-19, pois, embora não seja um marcador específico para a doença, está associado à gravidade e ao risco de mortalidade.

\section{REFERÊNCIAS}

GAFFENY, PJ. Breakdown products of fibrin and fibrinogen: molecular mechanisms and clinical implications. Journal of clinical pathology, London, ano 1980, v. 33, ed. 14, p. 10-17, 1 jan. 1980.

HENRY, Brandon Michael et al. Hyperinflammation and derangement of renin-angiotensin-aldosterone system in COVID-19: A novel hypothesis for clinically suspected hypercoagulopathy and microvascular immunothrombosis. Clin Chim Acta, [s. 1.], v. 507, p. 167-173, 26 abr. 2020. DOI 10.1016/j.cca.2020.04.027. Disponível em: https://pubmed.ncbi.nlm.nih.gov/32348783/. Acesso em: 7 nov. 2021.

MORENO, G et al. Revisión sistemática sobre la utilidad pronóstica del dímero-D, coagulación intravascular diseminada y tratamiento anticoagulante en pacientes graves con COVID-19. Medicina Intensiva , Tarragona, España, ano 2021, v. 45, p. 42-55, 17 jun. 2021. DOI https://doi.org/10.1016/j.medin.2020.06.006. Disponível em: https://pesquisa.bvsalud.org/portal/resource/pt/ibc190824. Acesso em: 15 out. 2021.

PILLAI, Presaas et al. COVID-19 and Major Organ Thromboembolism: Manifestations in Neurovascular and Cardiovascular Systems. J Stroke Cerebrovasc Dis, [s. 1.], v. 30, ed. 1, jan. 2021. DOI 10.1016/j.jstrokecerebrovasdis.2020.105427. Disponível em: https://pubmed.ncbi.nlm.nih.gov/33137615/. Acesso em: 5 nov. 2021.

ROSTAMI, Mehrdad; MANSOURITORGHABEH, Hassan. D-dimer level in COVID-19 infection: a systematic review. Expert Review of Hematology, [s. 1.], ano 2020 , v. 13 , ed. 11 , p. $1265-1275,12$ out. 2020 . DOI 10.1080/17474086.2020.1831383. Disponível em: https://pubmed.ncbi.nlm.nih.gov/32997543/. Acesso em: 15 out. 2021.

SAMKARI, Hanny Al et al. COVID-19 and coagulation: bleeding and thrombotic manifestations of SARS-CoV-2 infection. Blood, [s. 1.], v. 136, ed. 4, p. 489-500, 23 jul. 2020. DOI 10.1182/blood.2020006520. Disponível em: https://pubmed.ncbi.nlm.nih.gov/32492712/. Acesso em: 15 out. 2021.

SUBRAMANIAM, Saravanan; SCHARRER, Inge. Procoagulant activity during viral infections. Frontiers In Bioscience, [s. 1.], ano 2018, ed. 23, p. 1060-1081, 1 jan. 2018. DOI 10.2741/4633. Acesso em: 5 nov. 2021.

YU, Hai-Han et al. D-dimer level is associated with the severity of COVID-19. Elsevier, [s. 1.], v. 195, 27 jul. 2020. DOI 10.1016/j.thromres.2020.07.047. Disponível em: https://pubmed.ncbi.nlm.nih.gov/32777639/. Acesso em: 15 out. 2021.

ZHANG, Haibo et al. Angiotensin-converting enzyme 2 (ACE2) as a SARS-CoV-2 receptor: molecular mechanisms and potential therapeutic target. Intensive Care Med, [s. 1.], ano 2020, v. 46, ed. 4, p. 586-590, 3 mar. 2020. DOI https://doi.org/10.1007/s00134-02005985-9. Disponível em: https://pubmed.ncbi.nlm.nih.gov/32125455/. Acesso em: 5 nov. 2021.

ZHANG, Litao. D-dimer levels on admission to predict inhospital mortality in patients with Covid-19. Journal of Thrombosis and Haemostasis , [S. 1.], ano 2020, v. 18, n. $6, \quad$ p. 1324-1329, 19 abr. 2021. DOI https://doi.org/10.1111/jth.14859. Disponível em: https://onlinelibrary.wiley.com/doi/full/10.1111/jth.1485 9. Acesso em: 5 nov. 2021.

IZCOVICH, Ariel et al. Prognostic factors for severity and mortality in patients infected with COVID-19: A systematic review. Plos One, [s. 1.], v. 15, ed. 11, 17 nov. 2020. DOI 10.1371/journal.pone.0241955. Disponível em: https://pubmed.ncbi.nlm.nih.gov/33201896/. Acesso em: 15 out. 2021. 


\section{V.10 N.1 (2022) ISSN: 2317-434X}

fisiopatología de la lesión pulmonar aguda. Paralelismo con la sepsis. Medicina Intensiva , [s. 1.], v. 32, ed. 6, 2 ago. 2008. DOI 10.1016/S0210-5691(08)70958-6. Disponível em: https://www.sciencedirect.com/science/article/abs/pii/S0 210569108709586. Acesso em: 5 nov. 2021.

RAJA, Ali S et al. Evaluation of Patients With Suspected Acute Pulmonary Embolism: Best Practice Advice From the Clinical Guidelines Committee of the American College of Physicians. American College of Physicians, [s. 1.], 3 nov. 2015. DOI 10.7326/M14-1772. Disponível em: https://www.acpjournals.org/doi/pdf/10.7326/M141772. Acesso em: 5 nov. 2021.

GIANNIS, Dimitrios et al. Coagulation disorders in coronavirus infected patients: COVID-19, SARSCoV-1, MERS-CoV and lessons from the past. Journal of Clinical Virology, [s. 1.], v. 127, 9 abr. 2020. DOI 10.1016/j.jcv.2020.104362. Disponível em: https://pubmed.ncbi.nlm.nih.gov/32305883/. Acesso em: 15 out. 2021. 